\title{
Somatic Stimuli, Spinal Pathways, and Size of Cutaneous Fibers Influencing Unit Activity in the Medial Medulary Reticular Formation
}

\author{
K. L. CASEY \\ Department of Physiology, The University of Michigan, Ann Arbor, Michigan, 48104
}

Received April 4, 1969

Analysis of unit activity in the medial medullary reticular formation of unanesthetized, decerebrate and decereballate cats showed that: (i) $66 \%$ of the units responding to natural somatic stimuli were influenced only by firm pinching of skin folds or by heavy pressure over some part of their receptive fields; (ii) $86 \%$ of the units responding to electrical stimulation of cutaneous nerves required volleys containing both $\mathrm{A}$ and $\mathrm{A}$-delta fibers for maximum response; (iii) unit responses to nerve volleys including A-delta fibers could be maintained above $70 \%$ of maximum levels even though anodal polarization of the nerve had produced differential block of the larger A fibers; (iv) somatically evoked potentials and unit discharge could be markedly reduced or eliminated by section of the ventolateral spinal cord quadrants, but high cervical section of dorsal and dorsolateral fascicies had no effect; and $(v)$ evoked potentials and unit discharge could be elicited by stimulation in the dorsomedial midbrain tegmentum and by stimulation of the caudal, but not rostral, cut end of the dorsal columns sectioned between $\mathrm{C} 1$ and $\mathrm{C} 2$. These findings suggest that a neural population in the medial medullary reticular formation is part of a system subserving responses to noxious stimuli.

\section{Introduction}

Within the past several years, a number of studies have provided information about primary cutaneous afferents which may be important for the detection of noxious somatic stimuli $(6,11,14,17,19-22,29,33)$. Although a few unmyelinated fibers have been found to respond exclusively to strong thermal or mechanical stimuli (20-22), a relatively large proportion of the finely myelinated A-delta fibers studied by Burgess and Perl (6) and by Perl (33) were excited only by mechanical stimuli which could reasonably be considered noxious.

There is a somewhat smaller body of neurophysiological information concerning the possible CNS pathways and mechanisms which may subserve motor or sensory functions in response to intense somatic stimuli.

1 This work was supported by grant NB-06588 from the National Institute for Neurological Diseases and Stroke, U. S. Department of Health, Education, and Welfare. The technical assistance of Barbara Dorenkamp Meinershagen and Evelyn Lewis is gratefully acknowledged. 
Neurons responding exclusively or differentially to noxious somatic stimuli have been found in the medial and posterolateral thalamus of awake ( 8 , and anesthetized animals (34,30). Some spinal interneurons and ascending spinal pathways also have some properties suggestive of a nociceptive function $(23,29)$.

Recent anatomical $(2,4,28)$ and plysiological (3) observations suggest that the medial medullary reticular formation in the region of the nucleus gigantocellularis (27) may form part of a nociceptive system. The medial bulboreticular area receives somatosensory input via the ventrolateral qualrant of the spinal cord $(3,4,28)$, forms part of the ascending input to the medial thalamus (3), and is a source of reticulospinal projections $(25,31$, $42)$. Some of these bulboreticular neurons have axons projecting in both rostral and caudal directions $(25,37)$. Both stimulation $(26,41)$ and unit analysis $(25,42)$ have revealed some properties of the reticulospinal projections. In the decerebrate cat, Wolstencroft (42) found that a majority of reticulospinal neurons responded only to heavy mechanical stimuli, a property also observed by Oscarsson and Rosén (32) in a study of reticulocerebellar fibers. More recently, Burton ( 7 ) has reported that, in the intact, anesthetized or decerebrate cat, most of the units recorded from the tegmental field just lateral to the medial bulboreticular formation responded only to intense mechanical or thermal stimulation of the face.

In addition to receiving input via the ventrolateral spinal cord and responding maximally or exclusively to intense somatic stimuli, a neural population with the capacity of serving nociceptive functions might also be expected to receive a major portion of its cutaneous input from the smaller diameter cutaneous afferents. A medial bulboreticular population with these properties is found in the unanesthetized, decerebrate cat and is described in this report.

\section{Methods}

Adult cats were anesthetized with ether during the decerebration procedure. Following occlusion of the common carotid arteries, the midbrain was transected immediately rostral to the superior colliculus, and ether anesthesia was discontinued. A high basilar artery ligation was performed on some cats prior to the transection in order to limit blood loss. The entire cerebellum was then removed by suction and, either at this point or later in the experiment, the dorsal columns were sectioned bilaterally between $\mathrm{C} 1$ and $\mathrm{C} 2$. The medulla and cervical cord were protected by polyethylene sheets and frequent, liberal applications of warm saline solution or mineral oil. Rectal temperature and femoral arterial blood pressure were continuously monitored in all animals. Following surgery, the cats were paralyzed with gallamine (Flaxedil) or decamethonium bromide (Svrn- 
curine) administered intravenously as needed throughout the experiment. Respiration was artificially maintained at $18-25$ strokes $/ \mathrm{min}, 30-35 \mathrm{ml}$ tidal volume. The percentage of $\mathrm{CO}_{2}$ in the expired air was monitored in about half of the animals ( 15 cats) with a calibrated infrared $\mathrm{CO}_{2}$ analyzer. In some cats, bilateral pneumothorax was performed in order to reduce respiratory movements. Throughout these experiments, it was observed that spontaneous and evoked unit activity in the bulboreticular formation was regularly recorded only from preparations with mean arterial pressures above $85-90 \mathrm{~mm} \mathrm{H} \mathrm{g}_{\mathrm{g}}$ and with expired $\mathrm{CO}_{2}$ levels in the range of $3-6 \%$. Indeed, the most sensitive indicator of the physiological status of the preparation seemed to be the frequency with which unit activity was encountered.

An array of four stimulating electrodes was stereotaxically positioned in the dorsomedial tegmentum of the midbrain a few millimeters posterior to the intercollicular level. The middle pair of this array was directed toward the region of the central tegmental tract, a region shown by others (25) to contain the axons of bulboreticular neurons with ascending connections. An additional purpose of midbrain stimulation was to identify those neurons receiving supramedullary input and thus help to distinguish the action potentials of spinoreticular axons from those of neurons in or near the medial medullary reticular formation. Any pair of electrodes in the array could be used for stimulation with either electrode as cathode.

Bipolar silver-silver chloride hook electrodes with a separation of 3-5 $\mathrm{mm}$ were used for stimulation of either the sural or superficial radial nerves and the dorsal columns. Either the rostral or caudal cut ends of the dorsal columins were lifted from the underlying spinal cord and onto the electrodes for stimulation. The dissected fascicles were also separated from the cord by a polyethylene sheet as additional protection against current spread. As a final check on the locus of effective stimulation, constant current pulses were applied to the cord immediately beneath the elevated dorsal column fascicles.

Anodal polarization of the peripheral nerves was performed according to the technique described elsewhere (9).

The natural stimuli routinely tested included clicks delivered through a loudspeaker; movement of hairs; touching or lightly tapping the skin with a blunt probe; gentle, steady pressure displacing subcutaneous tissue; joint movement; localized, radiant heat; pinching of skin folds; and heavy pressure applied over bony prominences. Joint movement was performed manually, care being taken to manipulate all adjacent joints in an effort to localize the effective stimulus. In some instances, it was not possible to determine the effectiveness of passive joint movement since pressure on adjacent cutaneous and subcutaneous tissue influenced unit activity. A small 
disc thermistor was used to measure skin temperature during the application of focused radiant heat from a high-intensity lamp. Skin folds were lifted from the underlying soft tissue and pinched either by hand or with a pair of pincers with one side connected to a spring-loaded potentiometer. The output of the potentiometer was calibrated with standard scales. Initial displacement of the pincers required $600 \mathrm{~g}$, and complete displacement required $1.5 \mathrm{~kg}$; the voltage output of the transducer was linearly related to force applied within this range. The flat edges of the pincer probes in contact with the skin had an area of $7 \mathrm{~mm}^{2}$. In many instances, the threshold for excitation of a single bulboreticular unit exceeded the range of the pincers; more intense mechanical stimuli were then delivered manually and usually consisted of blunt, heavy pressure over bony prominences. Overt tissue damage was avoided.

Stainless steel microelectrodes were prepared and tested in a manner previously described (13) and connected to conventional recording equipment. The amplified action potentials were led into a pulse generator with a Schmitt trigger input so that the peak of each action potential produced a standard pulse which was displayed on a pen writer and on the oscilloscope with the original recording. This technique was used only with spikes well above noise level and with nearly constant amplitudes during the period of observation. Spike amplitude and pulse triggering levels were frequently checked, especially during the application of intense stimuli where small increases in blood pressure might alter brain-electrode relationships $(8,34)$ and produce changes in unity activity or spike amplitude.

Responses to electrical stimuli were analyzed by constructing poststimulus histograms from filmed records. Except for stimuli delivered during anodal polarization, at least 30 stimuli of equal strength were used for each histogram. The peak amplitudes of both $\mathrm{A}$ and A-delta waves were measured as stimulus strength increased; during anodal polarization, however, changes in compound action potential amplitude sometimes permitted the analysis of only ten stimuli at a stable compound action potential amplitude. In any case, a chi-square test was used to determine whether the poststimulus spike distribution differed significantly $(p \leq .001)$ from the distribution expected without stimulation. Natural stimuli were considered to affect unit discharge if the responses satisfied the McNemar test for the significance of change $(p \leq 0.01)(40)$.

The electrode position along each penetration was electrolytically markerl at one to three sites and stained with the Prussian blue method (15). Frozen sections of $40 \mu$ thickness were stained by the Nissl method and used for reconstructing each electrode track and identifying the sites of recorded unit activity.

Terminology. Throughout this paper, the term "A wave" or "A fibers" 
will refer to all peripheral nerve fibers with conduction velocities above the A-delta range (approximately 24 meter/sec). The term "A-delta" wave or fiber will have its usual meaning.

\section{Results}

Ascending Spinal Pathways. As reported by others (3), electrical stimulation of cutaneous nerve evoked slow wave and unit activity throughout the medial bulboreticular region coextensive with the nucleus gigantocellularis (NGC). Bilateral section of the dorsal and dorsolateral columns between $\mathrm{C} 1$ and $\mathrm{C} 2$ had no effect on the latency or amplitude of these potentials. However, a unilateral high cervical ventrolateral cordotomy reduced the response amplitude, and a bilateral ventrolateral section climinated the response. This finding is in accord with the anatomical $(4,28)$ and physiological (3) evidence that the principal somatic input to the medial bulboreticular formation is via the ventrolateral rather than dorsomedial somatosensory pathways.

Further confirmation of this point was obtained by stimulation of the rostral cut end of the dorsal columns. As shown in Fig. 1, stimulation of

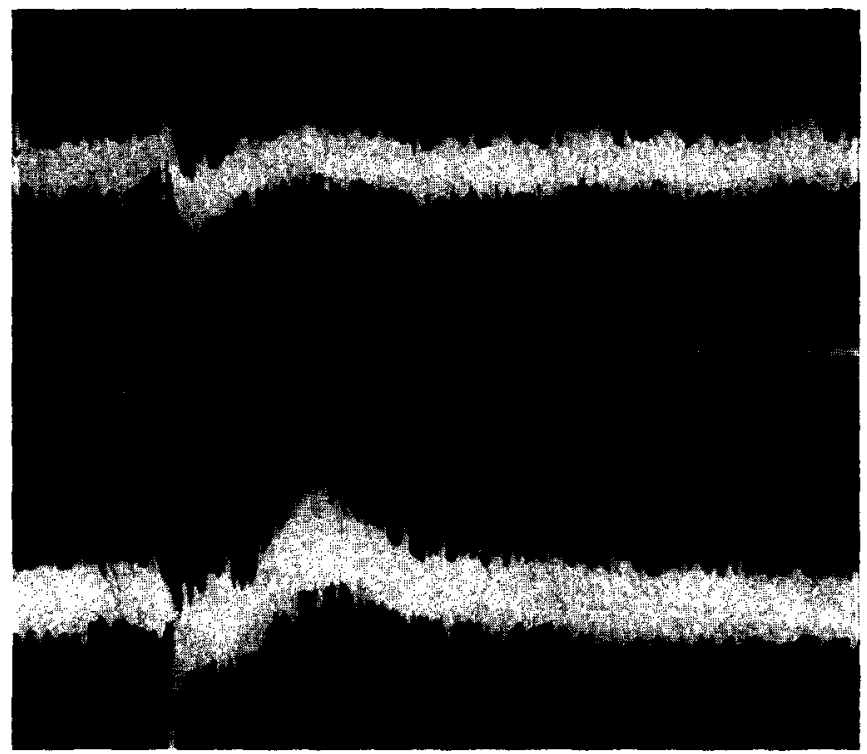

FIG. 1. Dorsal column projection to the medial medullary reticular formation. Dorsal columns sectioned between C1-C2. Upper. Stimulation of rostral cut end (250 $\mu \mathrm{amp}, 0.2 \mathrm{msec}$ ) evokes only short latency positive potential which increased in amplitude as medullary recording electrode approached the medial lemniscus. Unit was not driven by this stimulus. Lower. Same stimulus applied to caudal cut end evokes longer latency negative wave and discharge of units. Adequate stimulus for unit discharge was intense mechanical stimulation of either hind limb. Calibration: $200 \mu \mathrm{v}, 10 \mathrm{msec}$. 
the rostral cut end of the dorsal columns failed to evoke a field potential or unit discharge in the NGC. Stimulation of the caudal cut end of the dorsal columns, however, consistently evoked both slow potential and unit discharge (Fig. 1) in the NGC region (34 of 73 units tested). Response latencies ranged from 8 to $15 \mathrm{msec}$. These responses were not obtained by stimulation of the underlying cord and thus presunnably reflect the activation of dorsal column collaterals to spinoreticular neurons.

Direct Descending Pathways. Previous anatomical $(5,30,37)$ and physiological $(3,25)$ work demonstrated ascending connections from the medial bulboreticular formation. In an attempt to identify these elements by antidromic stimulation, an array of four stimulating electrodes was placed in the dorsal tegmentum at an intercollicular level. The medial pair of the array was positioned in the region of the central tegmental fasciculus or in the dorsomedial portion of the midbrain reticular formation. Bipolar stimulation was used, and any electrode in the array could be used as the stimulating cathode.

Midbrain stimuli of $0.1-1.0 \mathrm{mamp}$ and $0.2 \mathrm{msec}$ duration were routinely delivered at $1 / \mathrm{sec}$ during microelectrode exploration in order to excite units which were not spontaneously active. As the microelectrode traversed the medial medulla, midbrain stimulation evoked short latency, complex field potentials, the earliest phase of which attained maximum amplitude in the region of the NGC (Fig. 2). The shortest latency negative waves faithfilly followed repetitive midbrain stimulation up to 200 pulse sec. At higher stimulus frequencies (to $500 / \mathrm{sec}$ ), their amplitudes progressively decreased to approximately one-half of the control value but continued to follow each stimulus. Unit discharges were never observed to occur during the early negative potentials. The second, smaller negative waves would not follow repetitive midbrain stimulation in excess of $100 / \mathrm{sec}$; in fact, stimulus rates of $50 / \mathrm{sec}$ were often associated with a failure of discharge. In further contrast with the early negative potential, unit discharge was frequently associated with the second wave, especially when the recording electrode was in the NGC (Fig. 2).

A total of 164 medial bulboreticular units was tested for response to midbrain stimulation; of these, 89 responded at latencies ranging from $1.2-3.8 \mathrm{msec}$ and none followed repetitive stimuli in excess of $20 / \mathrm{sec}$. No units were observed to respond to rapid repetitive stimuli or at latencies consistent with antidromic activation. During the course of exploration. single shock or repetitive midbrain stimulation was never observed to inhibit spontaneous or evoked unit discharge.

While midbrain stimulation failed to detect those bulboreticular neuron: with direct ascending connections, the presence of a descending facilitatory system was confirmed (25), and the short latency unit responses provicled 


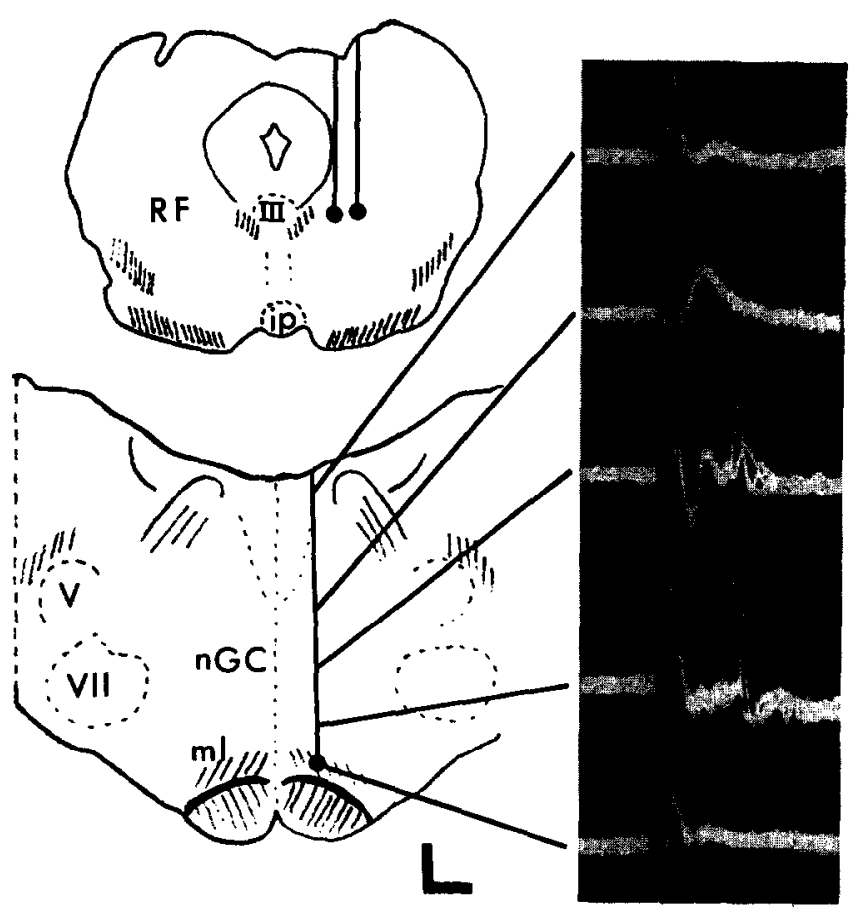

FIG. 2. Midbrain projection to the medial medullary reticular formation. Linc drawings show location of the effective bipolar stimulating electrodes (top) and recording microelectrode (bottom). Stimulation ( $300 \mu \mathrm{amp}, 0.2 \mathrm{msec}$ ) in the region of the central tegmental fasciculus. Multiple sweeps show unit driving and short latency negative waves with maximum amplitude near the ventral part of the nucleus gigantocellularis. Abbreviations. RF, reticular formation; ip, n. interpeduncularis; $\mathrm{ml}$, medial lemniscus; nGC, $\mathrm{n}$. gigantocellularis; III, V, VII, nuclei of oculomotor, trigeminal, and facial nerves.

assurance that these units were not recorded from the axons of spinoreticular neurons. The great majority $(87 \%)$ of the 264 units studied were initially and predominantly negative in polarity and had action potential durations over $0.5 \mathrm{msec}$; this observation lends further support to the suggestion that these units were recorded near the soma-dendritic region of the neuron (18).

Natural Somatic Stimuli. The natural somatic stimuli were divided into broad categories : intense and innocuous. Innocuous stimuli were of such an intensity that they never elicit withdrawal reflexes in the lightly anesthetized or unanesthetized, decerebrate cat and do not provoke behavioral reactions indicative of pain in the intact, awake animal. Stimuli classified here as "intense" include all mechanical stimuli delivered with the calibrated pincers as well as stronger, manually applied stimuli such as heavy pressure over 
bony prominences. Stimuli just sufficient to displace the pincers were moderately painful when applied to the back of the experimenter's hand, but did not consistently elicit withdrawal behavior in the unanesthetized intact or decerebrate cat; at higher intensity (above $1 \mathrm{~kg}$ ) withdrawal responses were consistently elicited. It is because of the uncertainty about the behavioral or sensory consequences of the stimuli that the term "intense stimulus" was used. Many of the effective intense stimuli used, however, could reasonably be considered noxious.

The functional distribution of NGC units completely tested for responses to natural somatic stimuli is shown in Fig. 3 and Table 1. None of these responded to auditory stimuli, and nearly one-half (49\%) failed to respond to any somatic stimulus tested. Unresponsive units were often found within $200 \mu$ to $300 \mu$ of units responding to gentle tapping or to heavy mechanical stimuli.

Innocuous Stimuli. A much smaller group of units (17.6\% of those tested) were found to respond only to innocuous stimuli. Intense stimuli, well above the threshold for activation of these units, did not appreciably increase the frequency or duration of discharge. Passive movement of joints was the only effective stimulus for five (15\%) of these. In each case, only extension or flexion of proxinal joints was effective for any given limb but
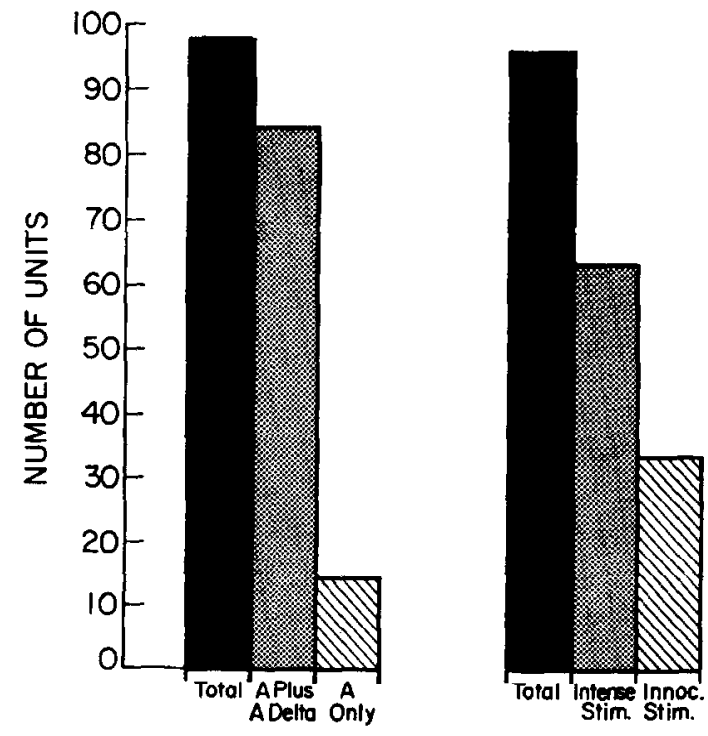

FIG. 3. Functional distribution of responsive bulboreticular units. The majority of responsive units required both $A$ and $A$-delta fibers for maximal response to electrical stimulation (left) and were influenced only by intense mechanical stimuli over some part of their receptive field (right). 
TABLE 1

Distribution of All Medial Bulboreticular Units Studied in Terms of Their Responses to Electrical Stimulation of Cutaneous Nerve and to Natural Mechanical Somatic Stimuli ${ }^{a}$

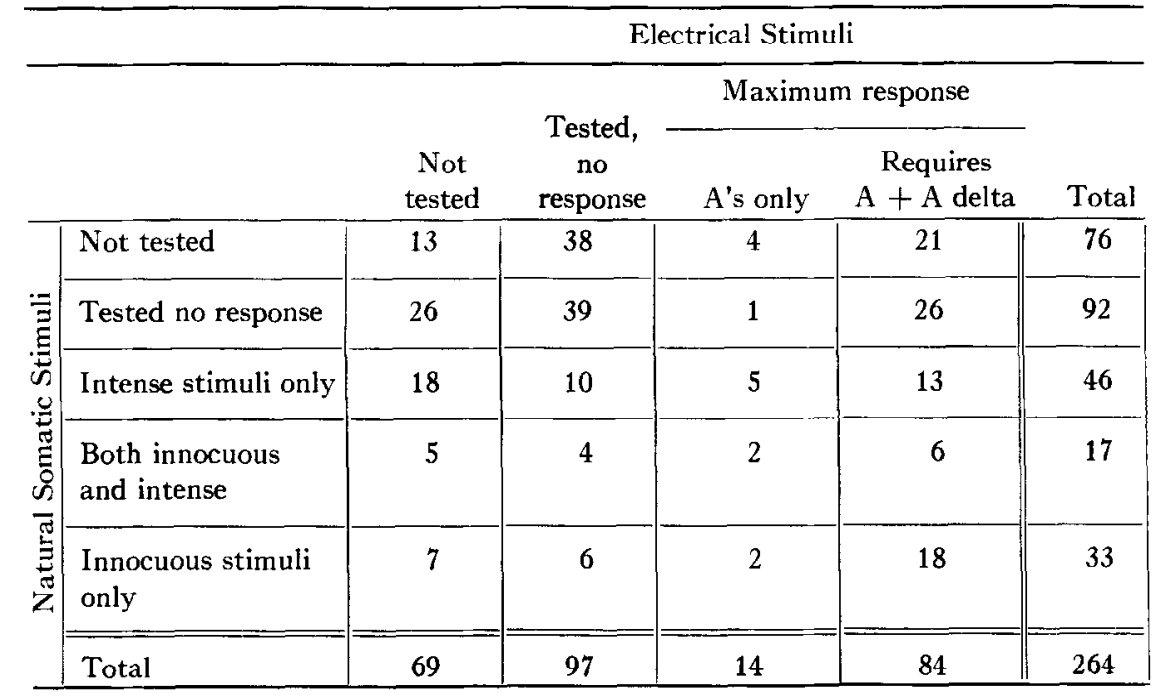

${ }^{a}$ Units responding to both innocuous and intense natural stimuli responded only to intense stimuli over some part of their receptive field.

movement of more than one limb could influence unit discharge. No attempt was made to locate the receptor sites by dissection, but squeezing the muscles acting across the joint or light pressure over the adjacent soft tissues was never effective. Manipulation of neighboring joints was always tested in order to determine the effective locus of stimulation.

The remainder of the units responding only to innocuous stimuli were influenced either by mild pressure over soft tissue areas (19 units) or by touching or tapping the skin (9 units). The former group responded only when the mechanical displacement involved subcutaneous structures; the effective stimulus may, therefore, involve afferents from muscle as well as other subcutaneous structures. Indeed, manipulation of joints was effective in six of these cases although the effective movement was never uniquely correlated with the action of a particular muscle group. In contrast with this group, those units responding to superficial skin stimulation could be driven by light pressure or touch to skin areas overlying bone as well as soft tissue; hair movement alone was not effective. In most cases, it was possible to elicit responses by tapping a fold of skin with a blunt probe. Joint movement also appeared to be an effective stimulus for three of these units, but there is less certainty in this case since skin stimulation 
necessarily accompanied joint movement and there was no instance in which the separate effects were restricted to different limbs.

Intense Stimuli. Perhaps the most striking finding in this study relates to the relatively large number of units responding exclusively to heavy mechanical stimuli over some body area (63 units). Of this group, 46 (73\%) responded exclusively to such stimuli; no somatic area was found for which clearly innocuous stimuli were effective. The remainder (17 units) responded only to strong stimulation over some area (e.g., one or two limbs), but were influenced by clearly innocuous stimuli over other areas as well. Figure 4 shows the anatonical distribution of the 63 units. Units responding only to innoctous stimuli, as well as unresponsive units, were found scattered throughout this same medial bulboreticular area.

The pincers were routinely used to pinch skin folds in testing these responses. In some cases, pinching a fold of skin was ineffective and heavy pressure over bone was required to elicit a response. Overt damage to the skin was avoided, however, to allow subsequent testing of other units. Radiant heating of the skin to $50-60 \mathrm{C}$ was tested for 18 of these units, and no responses were observed.

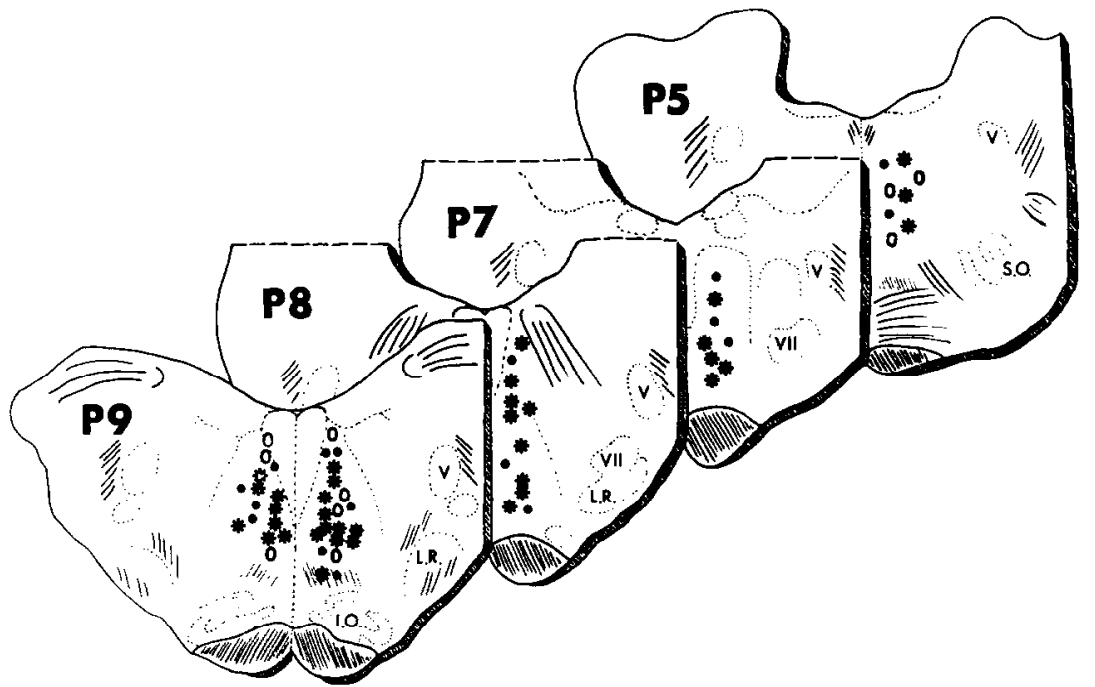

FIG. 4. Anatomical distribution of units influenced only by intense mechanical stimuli over some part of their receptive field (levels $P$ 5-9). Asterisks: units excited; intense stimuli required throughout receptive field. Open circles: same as with asterisks, but units were inhibited. Black dots: units either excited or inhibited unly by intense stimuli over some part of receptive field and influenced by innocuons stimuli elsewhere. Abbreviations: IO, inferior olivary nucleus; $\mathrm{SO}$, superior olivary nucleus; LR, lateral reticular nucleus; $v$, trigeminal; vii, facial. 
Migure 5 shows the type of discharge elicited by strong mechanical stim.uli. In all cases, the period of altered frequency lasted for the duration of ti:e stimulus and usually for 1-2 sec beyond stimulus termination. Excitatory responses were usually maximal at stimulus onset; no consistent "off" responses were observed. Figure 6 shows the responses of three different reticular units to graded intensities of skin fold pinching. The stimulusresponse relationship shown in this figure, however, was not observed in the majority of cases. Most commonly (32 of 40 units examined), the unit responded only to intense stimulation, but without a clear relationship to various intensities above threshold.
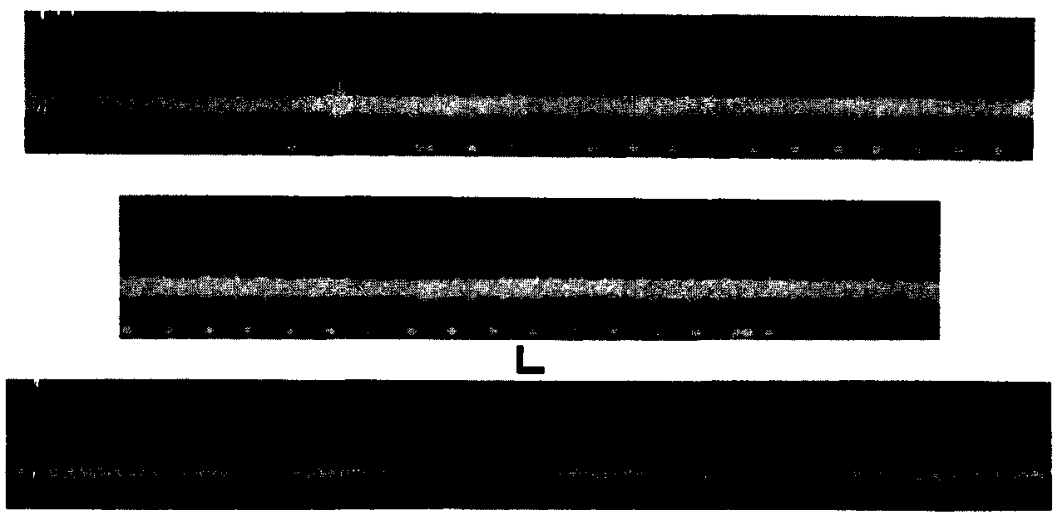

FIG. 5. Unit responses in nucleus gigantocellularis. Upper two traces show increa:ed firing during heavy pressure over contralateral tibia. Pulses at $10 / \mathrm{sec}$ signal stimulation period. Lower traces: four successive shocks to contralateral superficial radial nerve. Volley (not shown) included maximum amplitude $\mathrm{A}$ and $\mathrm{A}$-delta waves. Calibration: $20 \mathrm{msec}, 200 \mu \mathrm{r}$.

As in the case of units responding to innocuous stimuli. the receptive fiel. Is of the higher threshold neurons were large, usually covering an entire limb, more than one limb, or the whole body surface. Interaction between the receptive fields or even between different types of stimuli within the sarne field was also observed. Figure 7, for example, shows the inhibitory effect of intense stimulation applied to the same or opposite limb during continuous excitation by an innocuous stimulus. Definite interactions could not be demonstrated in the majority of cases, however, since the direction of effect on unit discharge was the same for the different stimulus categories and receptive fields.

Electrical Stimulation of Cutaneous Nerve. Only the contralateral superficial radial or sural nerves were tested in this phase of the study since additional dissection limited the testing of natural stimuli. Ninety-eight out of 195 units tested responded to electrical stimuli. The poststimulus histo- 


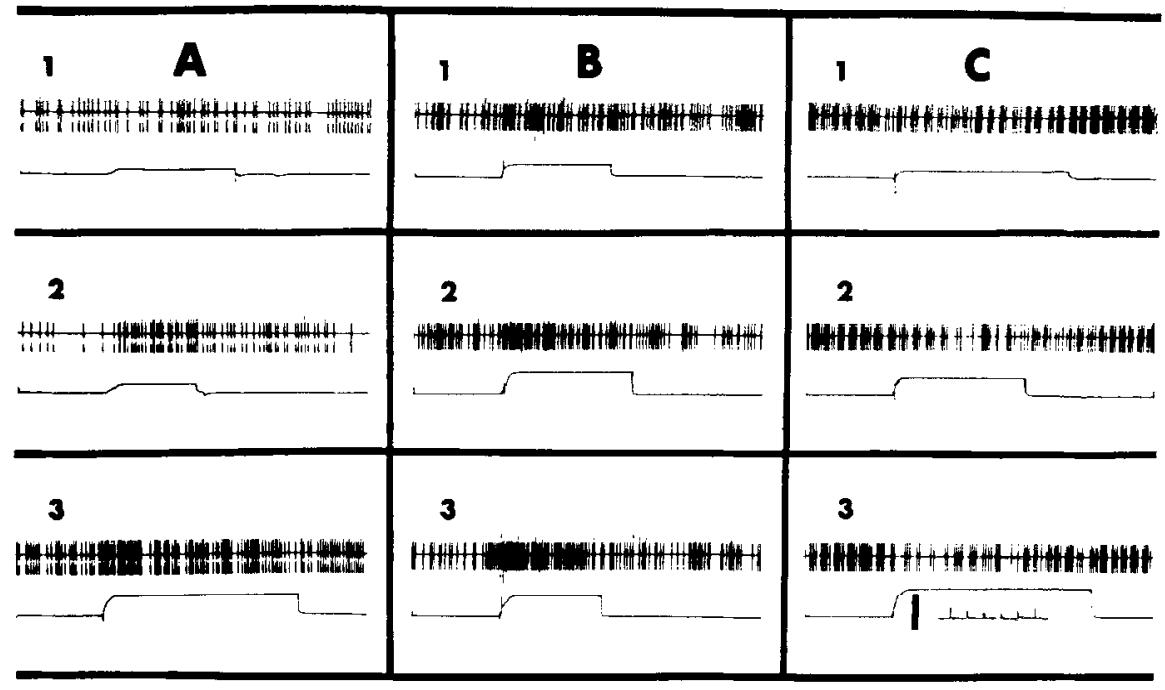

FIc. 6. Graded responses to intense mechanical stimuli. Pulsc gencrator output (top lines) triggered by units $\mathrm{A}, \mathrm{B}$, and $\mathrm{C}$. Pinch of skin fold registered by output of pincer potentiometer (lower lines). Increasing stimulus intensities (upper $\rightarrow$ middle $\rightarrow$ lower rows) produced increased excitatory $(A, B$,$) or inhibitory (C) re-$ sponses. Calibrations: $1.5 \mathrm{~kg} ; 1 \mathrm{sec}$.
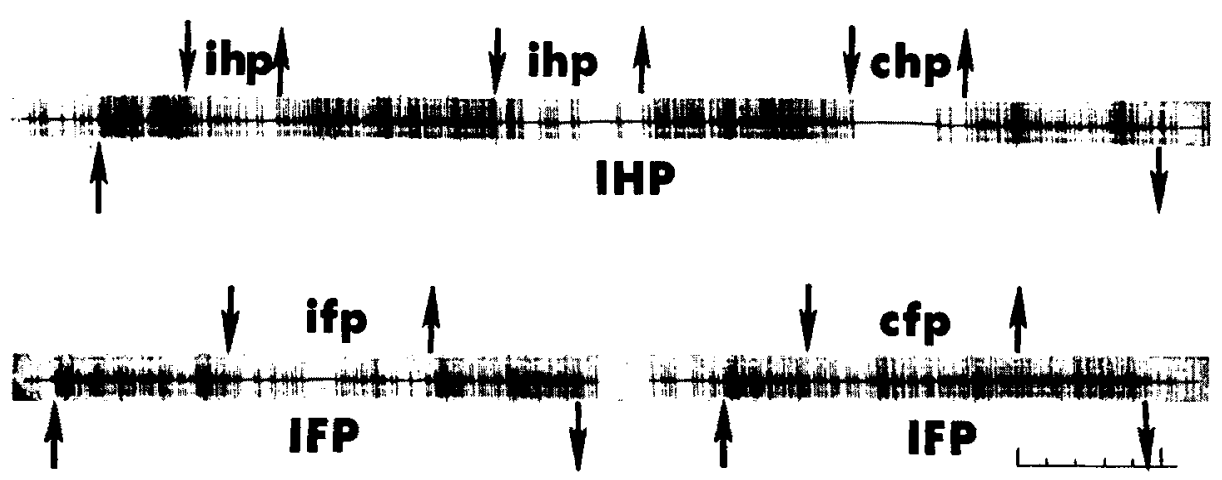

FIg. 7. Interaction between stimuli. Pulse generator output shows that strong pinch of skin folds of either hind limb (ihp, chp) inhibits the background discharge produced by continuous extension of ipsilateral ankle (IHP) (top line). Arrows indicate stimulus onset and offset. Excitation of same unit by ipsilateral forelimb (IFP) extenson was inhibited by strong skin pinch to that same limb (ifp) (lower left); similar stimulus to the contralateral forelimb (cfp) was ineffective llower right). Calibration : 1 isec. 
grams constructed in each case showed that all units responded within 30 msec and that the maximum probability of response occurred within 60 msec (Fig. 9). For all testing with electrical stimulation, the stimulus repetition rate was $1-2 /$ sec since preliminary observations revealed a lower response probability with repetition rates above $3-4 / \mathrm{sec}$.

The principal objective of this phase of the study was to determine the portion of the afferent fiber diameter spectrum evoking unit discharge. In order to quantify the responses, poststimulus histograms of the unit activity were constructed for several values of $\mathrm{A}$ and $\mathrm{A}$-delta wave amplitude. The average number of spikes per stimulus in each time bin was computed and summed over the entire poststimulus period (usually $140 \mathrm{msec}$ ), giving a measure of poststimulus probability. For comparing the relative effectiveness of different volleys, unit responsiveness was expressed as a percentage of the maximum poststimulus probability observed. Unit discharge was continuously monitored on a polygraph to assure that there were no gross changes in spontaneous firing during data acquisition.
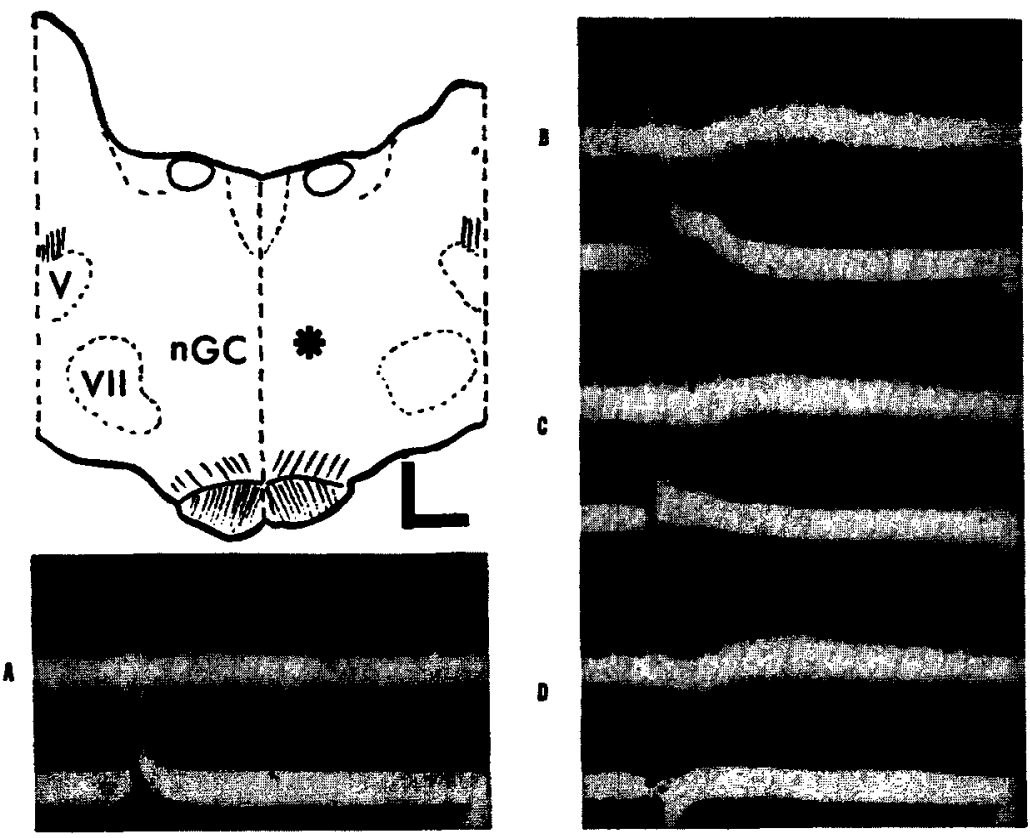

FIG. 8. Slow wave and unit responses in nucleus gigantocellularis during anodal polarization of peripheral nerve volley. Brain drawing shows position of recording microelectrode (asterisk). A-D, Superimposed sweeps of small positive and negativepositive units and slow potential (top traces) showing response as A-delta fibers (lower traces) are added (A-B) and persistence of response as larger fibers are blocked by anodal polarization (C-D). See text for further discussion. Calibration: $20 \mathrm{msec}, 200 \mu \mathrm{v}$ for top traces. 


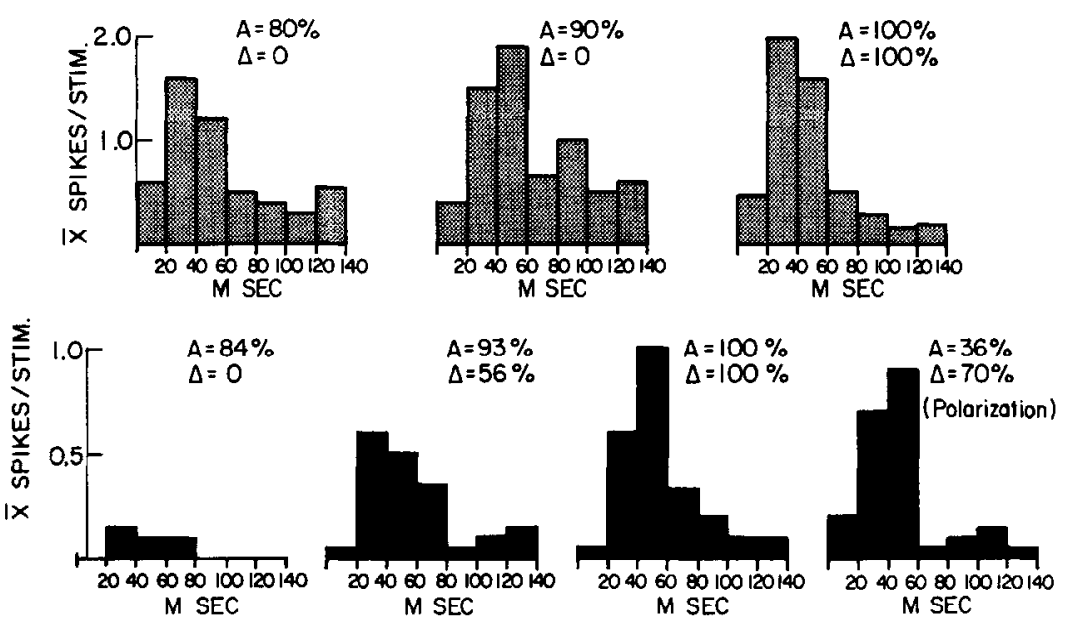

FIG. 9. Differences in responsiveness to $A$ and $A$-clelta volleys. Upper poststimulus histograms show little or no change in unit driving as A-delta fibers are added to the volley. Lower histograms show minimal effect of largc $\Lambda$ volley, but increasing response as A-delta fibers are added. Anodal polarization (lower right histogram) reveals the differential effect of the smaller fibers as some of the larger A fibers are blocked. Both units recorded from nucleus gigantocellularis.

It was first observed that, in the unanesthetized decerebrate cat, only stimuli above A-delta threshold evoked both slow potential and unit discharge in the NGC; full A volleys were ineffective (Fig. 8). However, 14 tinits responded at maximal or near maximal (75-80\%) poststimulus probabilities before the $\mathrm{A}$ wave had reached its maximum amplitude and before the $A$-delta wave appeared in the biphasic cutaneous nerve recording. Figure 9 shows the poststimulus histograms of one of these units before and after adding a full A-delta volley to the afferent discharge. As in each of these 14 cases, the addition of $\mathrm{A}$-delta fibers was without appreciable effect. An effective natural stimulus was determined for nine of these units; two responded to innocuous stimuli to the contralateral forelimb but were driven only by intense mechanical stimulation to other body areas, two were driven by innocuous stimuli throughout their receptive fields, and five responded only to intense somatic stimuli.

The remaining 84 units responded with maximal probability only to volleys in which both $\mathrm{A}$ and $\mathrm{A}$-delta waves were at maximum amplitude (Figs. 3 and 9). The addition of $C$ fibers had no effect on unit responses. These units varied in their degree of responsiveness to the larger diameter A fibers, but none responded with poststimulus probabilities above $50 \%$ of the maximum until the $\mathrm{A}$ wave was at least $75-80 \%$ of its maximum amplitude (Fig. 11). At this point, the A-delta wave could usually be 
detected; some A-delta fibers may have been excited at stimulus strengths just below this level, but were not detected in the biphasic nerve recording.

The above observations, however, did not distinguish between the effects of high threshold A fibers, total volley size, or a specific effect of A-delta fibers. Accordingly, anodal polarization was applied during stimulation in order to differentially block the large fibers. The interpretive and technical problems associated with this technique have been considered in greater detail elsewhere (9). During polarization, the composition of the volley is continuously changing; both large and small diameter fibers are being blocked and there are changes in the conduction velocities of the remaining
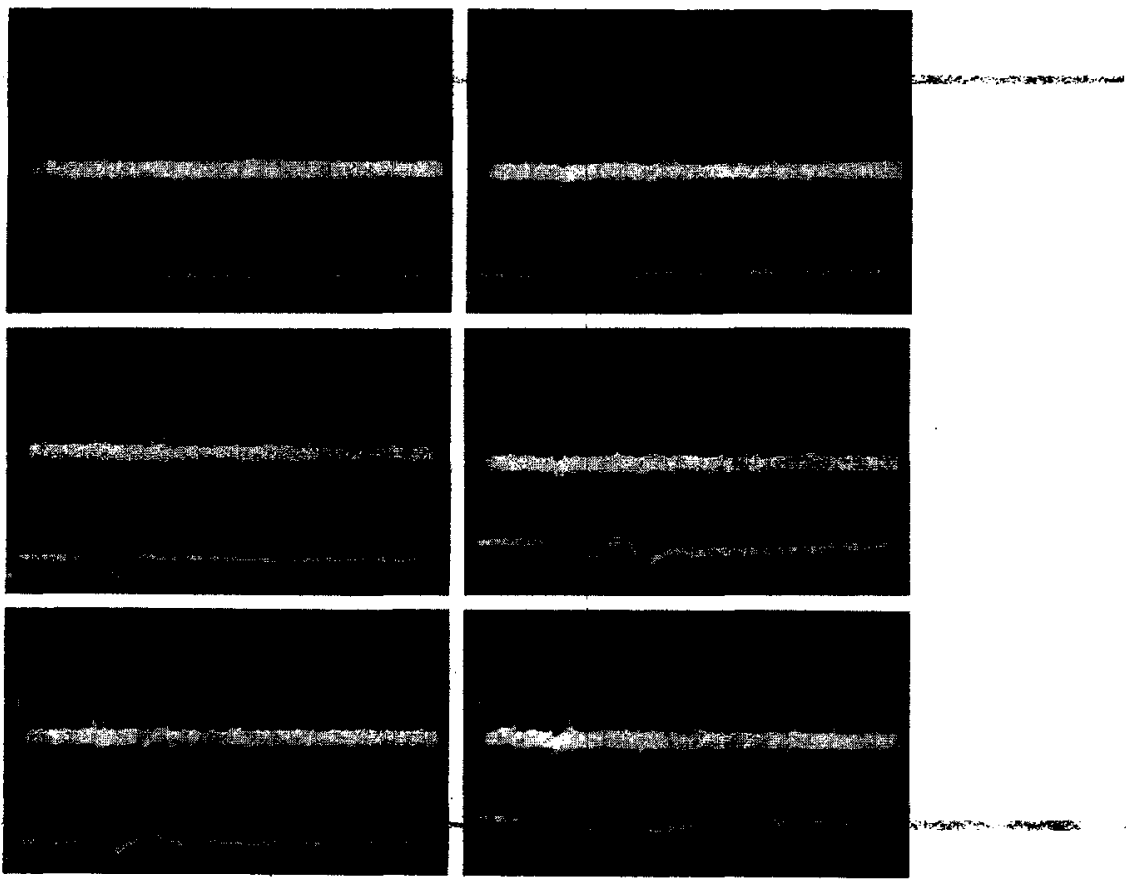

Fig. 10. Differential effect of A and A-delta fibers revealed by anodal polarization. Medial bulboreticular unit (dorsal border of nucleus gigantocellularis) begins to respond consistently (top traces) only as A-delta wave appears in the nerve record (lower traces) (A-D). Consistent responses are still obtained during anodal polarization (E-F) when some of the larger A fibers are blocked.

active fibers. These factors seriously limited the ability to obtain distinct response probabilities at each stage of block. Nevertheless, for 23 units, it was possible to achieve either a partial or a complete block of the $\mathrm{A}$ fibers while the A-delta afferents were relatively unaffected. Figures 8-11 show sample records and plotted data which indicate a differential effect 
of A-delta fibers on the responses of these units. If the increased poststimulus discharge were simply a function of $\mathrm{A}$ amplitude, partial polarization block of these fibers should have produced a marked decrease in unit response according to the stimulus-response curve obtained as A fibers were added to the volley (open circles, Fig. 11). A full A-fiber block would be expected to eliminate evoked unit discharge. However, the units continued to respond at or near the probability levels which might be expected if A-delta fibers were a major source of excitation. When apparently complete block was achieved (Fig. 8) some units continued responding. Because of the effects of anodal polarization, it is likely that the effective A-delta volley was not detected in the nerve recording in these cases (9).

The differential effect of small-diameter myelinated fibers is further emphasized in the plotted data of Fig. 11. The response curves of two of
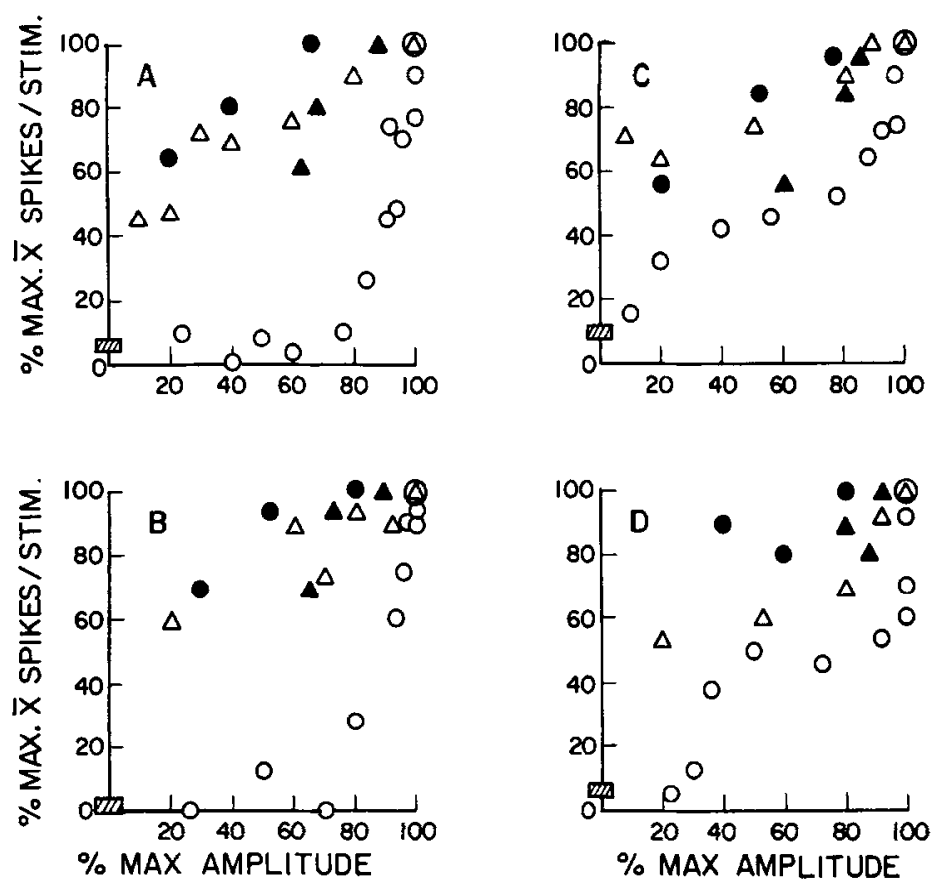

FIG. 11. Differential effect of $A$ and A-delta volleys. Coordinates indicate unit responses and compound action potential amplitudes as a percentage of the maximum observed during testing of each unit. Open circles and triangles indicate $A$ and A-delta waves, respectively, as volley size was increased before polarization. Filled symbols plot unit response as a function of these waves during anodal polarization of cutaneous nerve. A,B. Two units showing clearest differentiation of $A$ and A-delta effects. C,D. Two units which showed least differentiation among those tested. See text. 
these units (Fig. $11 \mathrm{~A}$ and $\mathrm{B}$ ) show the clearest differentiation obtained between $A$ and A-delta effects; during polarization, the responses followed the function described by the relative amplitudes of the A-delta, rather than the A waves. The same is true, at least for response levels near the maximum, in the examples shown in Fig. $11 \mathrm{C}$ and D. These two units showed the least clear differentiation between $\mathrm{A}$ and A-delta effects; they were driven at relatively low levels (below $50 \%$ of maximum) by $A$ volleys near $50 \%$ of maximum amplitude. With increasing stimulus strength, responsiveness rapidly approached the maximum levels as both $\mathrm{A}$ and A-delta fibers were added to the volley. However, response levels could be maintained above $70 \%$ of maximum even though anodal polarization had differentially reduced the A wave to nearly $50 \%$ of its maximum amplitude. The smaller myelinated fibers, then, do appear to contribute to the somatic input to these neurons although the above observations do not exclude the theoretically unlikely possibility that $\mathrm{A}$ afferents with thresholds in the $\mathrm{A}$ delta range were the first to be blocked by anodal polarization.

Relation of Responses to Natural and Electrical Stimuli. A direct correlation of responses to electrical and natural stimuli within the same receptive field cannot be made since most of the nerve strands were cut distally. Moreover, there was no assurance that surgical manipulation of these strands and repeated trials of polarization would not alter the character of the effective natural stimuli. These factors may account for some of the units which were affected by electrical, but not natural, stimuli and vice versa.

Table 1 shows that approximately one-half of the mechanosensitive units requiring A-delta fibers for maximum response were affected only by intense somatic stimuli over some part of their receptive field. Some degree of overlap between these categories might be expected since they each comprise a significant fraction of the responsive population. However, 18 units receiving A-delta input were affected only by innocuous stimuli, and five units influenced only by intense stimuli did not require A-delta fibers for maximum response.

\section{Discussion}

These results confirm previous anatomical $(2,4,28)$ and physiological (3) evidence that the medial medullary reticular formation in the region of nucleus gigantocellularis receives somatic input principally, if not entirely, via the ventrolateral spinal cord. Stimulation of the rostral cut end of a high cervical dorsal column section failed to evoke slow wave or unit discharge in the NGC region; antidromic stimulation of the dorsal columns, however, excited the spinoreticular projections to this area, presumably via the extensive collateral system from the dorsal fasciculi $(36,38)$. The effec- 
tiveness of this pathway may be attributed to the collaterals of smaller myelinated dorsal column fibers (16) or simply to the size and distribution of the large fiber volley within the spinal cord. There is also the possibility that spinoreticular neurons were discharged via presynaptic action on primary A-delta cutaneous afferents. Whatever the mechanism, the results of this study indicate some overlap between the dorsal column and ventrolateral somatosensory pathways at spinal. but not medial medullary, levels.

Stimulation in the dorsomedial midbrain tegmentum, in the region of the central tegmental fasciculus, failed to demonstrate those reticular neurons with ascending axons. Neurons of this type comprised less than $10 \%$ of the total population of reticular formation intracellular recordings reported by Magni and Willis (25). The reason for the relative difficulty in identifying these cells is not clear since there is ample anatomical evi dence $(5,30,37)$ for the existence of reticular neurons with axons ascending to midbrain levels or further rostrally. Brodal and Rossi (5) estimated that about one-third of the reticular formation neurons in this region are of this type. The midbrain stimuli in this study, however, did reveal the clirect excitatory influence from this area to medial bulboreticular neurons.

The principal finding is that, in the unanesthetized decerebrate cat, a large proportion of somatically driven medial bulboreticular neurons respond exclusively to heavy mechanical stimuli over all or part of their receptive field and respond with maximum probability only when a sample cutaneous volley includes A-delta fibers. Most of the cffective mechanical stimuli were sufficiently intense to provoke withdrawal reflexes in lightly anesthetized or decerebrate animals; while these observations suggest that these stimuli are noxious, behavioral studies will be required to test this possibility. These stimuli are, however, more intense than the effective natural stimuli reported by Bowsher, et al. (3) and Segundo et al. (39) in explorations of the medial bulbar reticular formation. It is possible that the observed differences are attributable to sampling variations in spite of the similarities in sample size and region of microelectrode exploration. Deterioration of respiratory or circulatory function seems unlikely to account for the discrepancy since monitoring of blood pressure and expired $\mathrm{CO}_{2}$ showed that both spontaneous and evoked unit activity are seen only when these physiological parameters are within normal limits. Moreover, many units responding to innocuous stimuli were affected only by intense stimuli in another part of the receptive field, and exclusively high-threshold cells were found in the same penetrations as those responding to touching. mild pressure, or tapping the skin.

The decerebration and removal of the cerebellum may alter the response properties of medial medullary reticular neurons and may also account for the high percentage of unresponsive cells and the lack of effective auditory 
input. Certainly there is ample evidence that descending influences from supramedullary structures can alter the excitability of primary afferents and interneurons in the spinal cord (24). The responses of bulboreticular neurons in the decerebrate cats may be strongly influenced by the action of descending pathways to the spinal cord or, more directly, to the medullary reticular formation itself. Removing the direct excitatory influence from the midbrain regions stimulated in this study may also affect the responses of medullary reticular units. The effects of surgery, however, would seem unlikely to produce a selective inhibition of elements highly sensitive to mechanical stimuli especially since, in many cases, this action would have to be restricted to a limited portion of the receptive field. High threshold mechanosensitive cells may, in fact, form an appreciable part of the medial bulboreticular population, for such units have been observed in medullary reticular areas of intact, anesthetized and decerebrate cats $(7,42)$ as well as in other regions of intact $(34,35)$, spinal $(23)$, and decerebrate preparations (32).

The smaller diameter, finely myelinated cutaneous afferents were predominantly effective in evoking bulboreticular activity. These results complement and extend the earlier observations on midbrain reticular formation responses to cutaneous $\mathrm{A}$-delta ( 1$)$ and $\mathrm{C}$-fiber volleys $(10,12)$. In the present study, the response latencies are consistent with the apparent lack of effect of $\mathrm{C}$ fiber stimulation on unit and evoked potential discharge. The differential effect of A-delta fibers, however, was revealed by partial or nearly complete block of the larger-diameter afferents, thus serving to distinguish between the effects of volley size and composition. Even those units receiving some input via $A$ fibers were primarily influenced by changes in A-delta amplitude. The site and mechanism of action of these finely myelinated fibers remain subjects for future investigation.

The adequate stimulus for these neurons is similar in some respects to that reported by Burgess and Perl (6) for A-delta fibers in cat and primate cutaneous nerve. Thus, many of these central cells are influenced by heavy mechanical stimuli, but not by intense, local heat. In comparison to units driven by touch or hair movement, the responses are slowly adapting. The sample of bulboreticular neurons, however, includes fewer deltasensitive elements responding to innocuous stimuli than might be expected from the proportion of highly sensitive A-delta afferents $(6,33)$.

The functional significance of these findings deserves further investigation. The current anatomical and physiological evidence suggest that this bulboreticular region is a nodal point through which cutaneous stimuli may influence both motor and, possibly, sensory function. Previous studies (26, 41) demonstrated the motor effects produced by stimulation of this region in awake, anesthetized, and decerebrate cats, but much remains to be learned 
about its possible role in sensory, motivational, or higher integrative functions. The results of studies in the decerebrate cat, at any rate, indicate that both behavioral and electrophysiological techniques should be used to further test the possibility that a neural population in the medial medullary reticular formation forms part of a nociceptive system. A more general function is suggested by the heterosensory convergence onto some neurons in this area of the intact cat $(3,39)$.

\section{References}

1. Amassian, V. E., and R. V. DeVito. 1954. Unit activity in reticular formation and nearby structures. J. Neurophysiol. 17: 575-603.

2. Busher, D. 1957. Termination of the central pain pathway in man : the conscious appreciation of pain. Brain $80: 606-621$.

3. Bowsher, D., A. Mallart, D. Petit, and D. Albe-Fessard. 1968. A bulbar relay to the centre median. J. Neurophysiol. $31: 288-300$.

4. BREAzile, J. E., and R. L. Kitchell. 1968. Ventrolateral spinal cord afferents to the brain stem in the domestic pig. J. Comp. Neurol. 133: 363-372.

5. Brodal, A., and G. F. Rossi. 1955. Ascending fibers in brain stem reticular formation of cat. A.M.A. Arch. Neurol. Psychiat. 74: 68-87.

6. Burgess, P. R., and E. R. PerL. 1967. Myelinated afferent fibres responding specifically to noxious stimulation of the skin. J. Physiol. London 190: 541562.

7. Burton, H. 1968. Somatic sensory properties of caudal bulbar reticular neurons in the cat (felis domestica) Brain Res. 11: 357-372.

8. CASEY, K. L. 1966. Unit analysis of nociceptive mechanisms in the thalamus of the awake squirrel monkey. $J$. Neurophysiol. $29: 727-750$.

9. CASEY, K. L., and M. Blick. 1969. Observations on anodal polarization of cutaneous nerve. Brain Res. 13: 155-167.

10. Collins, W. F., and C. T. Rannt. 1960. Midbrain evoked responses relating to peripheral unmyelinated or ' $\mathrm{C}$ ' fibers in cat. $J$. Neurophysiol. 23: 47-53.

11. Collins, W. F., F. E. Nulsen, and C. T. Randt. 1960. Relations of peripheral nerve fiber sine and sensation in man. Arch. Neurol. Psychiat. 3: 381-385.

12. Collins, W. F., and J. L. O'Leary. 1954. Study of a somatic evoked response of midbrain reticular substance. Electroencephalog. Clin. Neurophysiol. 6: 619-628.

13. Cuenod, M., K. L. Casey, and P. D. MacLean. 1965. Unit analysis of visual input to posterior limbic cortex. I. Photic stimulation. J. Neurophysiol. 28: $1101-1117$.

14. Douglas, W. W., and J. M. Ritchie. 1957. Non-medullated fibres in the saphenous nerve which signal touch. J. Physiol. London. 139: 385-399.

15. GREen, J. D. 1958. A simple microelectrode for recording from the central nervous system. Nature $182: 962$.

16. HäGGQvist, G. 1936. Analyse der Faserverteilung in einem Rückenmarkquerschnitt. Z. Mikroskop.-Anat. Forsch. 39: 1-34.

17. Heinbecker, P., G. H. Bishop, and J. O'Leary. 1933. Pain and touch fibers in peripheral nerves. A.M.A. Arch. Neurol. Psychiat. 29: 771-789.

18. Huber, D. H. 1960. Single unit activity in lateral geniculate body and optic tract of unrestrained cats. J. Physiol. London 150: 91-104. 
19. Hunt, C. C., and A. K. MCIntyre. 1960. An Analysis of fibre diameter and receptor characteristics of myelinated cutaneous afferent fibers in cat. $J$. Physiol. London 153 : 99-112.

20. IGGo, A. 1959. Cutaneous heat and cold receptors with slowly conducting (C) afferent fibres. Quart. J. Exptl. Physiol. 44: 362-370.

21. IGgo, A. 1960. Cutaneous mechanoreceptors with afferent C fibres. J. Physiol. London 152 : 337-353.

22. Iriuchijima, J., and Y. Zotterman. 1960. The specificity of afferent cutaneous C fibres in mamnals. Acta Physiol. Scand. $49: 267-278$.

23. Kolmodin, G. M., and C. R. Skoglund. 1960. Analysis of spinal interneurons activated by tactile and nociceptive stimulation. Acta Physiol. Scand. 50:337355.

24. LUNDBERG, A. 1964. Supraspinal control of transmission in reflex paths to motoneurons and primary afferents, pp. 197-219. In "Progress in Brain Research," vol. 12: "Physiology of Spinal Neurons," J. C. Eccles and J. P. Schadé [Eds.], Elsevier, Amsterdam.

25. MagNI, F., and W. D. Willis. 1963. Identification of reticular formation neurons by intracellular recording. Arch. Ital. Biol. 101: 681-702.

26. Magoun, H. W., and R. Rhines. 1946. An inhibitory mechanism in the bulbar reticular formation. J. Neurophysiol. 9 : 165-171.

27. Meessen, H., and J. Olzewski. 1949. "A Cytoarchitectonic Atlas of the Rhombencephalon of the Rabbit." Karger, Basel, Switzerland.

28. Mehler, W. R., M. E. Feferman, and W. J. H. Nauta. 1960. Ascending axon degeneration following anterolateral cordotomy. An experimental study in the monkey. Brain $83: 718-750$.

29. Mendell, L. M. 1966. Physiological properties of unmyelinated fiber projection to the spinal cord. Exptl. Neurol. $16: 316-332$.

30. Nauta, W. J. H., and H. G. J. M. Kuypers. 1958. Some ascending pathways in the brain stem reticular formation, pp. 3-30. In "Reticular Formation of the Brain," H. H. Jasper, et al. [Eds.], Little, Brown, Boston.

31. NyberG-HANSEN, R. 1965. Sites and mode of termination of reticulo-spinal fibers in the cat. J. Comp. Neurol. 124: 71-100.

32. Oscarsson, O., and I. Rosén. 1966. Response characteristics of reticulocerebellar neurons activated from spinal afferents. Exptl. Brain Res. 1: 320-328.

33. PERL, E. R. 1968. Myelinated afferent fibres innervating the primate skin and their response to noxious stimuli. J. Physiol. London 197: 593-615.

34. Pert, E. R., and D. G. Whitlock. 1961. Somatic stimuli exciting spinothalamic projections to thalamic neurons in cat and monkey. Exptl. Neurol. $3: 256-296$.

35. Poggio, G. F., and V. B. Mountcastle. 1960. A study of the functional contributions of the lemniscal and spinothalamic systems to somatic sensibility. Central nervous mechanisms in pain. Johns Hopkins Hosp. Bull. 106: 266-316.

36. Ramón y Cajal, S. 1909. "Histologie du Systeme Nerveux." Maloine, Paris.

37. Scheibel, M. E., and A. B. Scheibel. 1958. Structural substrates for integrative patterns in the brain stem reticular core, pp. 31-55. In "Reticular formation of the brain." II. H. Jasper et al. [Eds.], Little, Brown, Boston.

38. Scheibel, M. E., and A. B. Scheibel. 1968. Terminal axonal patterns in cat spinal cord. II. The dorsal horn. Brain Res. 9 : 32-58.

39. Segundo, J. P., T. Takenaka, and H. EnCabo. 1967. Somatic sensory properties of bulbar reticular neurons. J. Neurophysiol. 30 : 1221-1238. 
40. Sieged, S. 1956. "Non-Parametric Statistics for the Behavioral Sciences," pp. 63-67. McGraw-Hill, New York.

41. Sprague, J. M., and W. W. Chambers. 1954. Control of posture by reticular formation and cerebellum in the intact, anesthetized and unanesthetized and in the decerebrated cat. Am. J. Physiol. 176: 52-64.

42. Wolstencroft, J. H. 1964. Reticulospinal neurones. J. Physiol. London 174: 91-108. 